\title{
Trastocamiento de la salud en la cotidianidad de las mujeres embarazadas con pre-eclampsia*
}

\section{Health Disruption in everyday life of pregnant women with preeclampsia.}

Mtra. María de los Ángeles Torres Lagunas•

Dra. Flor Marlene Luna Victoria..

Dra. María Alberta García Jiménez....

\section{Resumen}

El presente estudio cualitativo, descriptivo, exploratorio, tiene el objetivo de describir y analizar la cotidianidad de las mujeres que presentan pre-eclampsia de una comunidad semiurbana del Municipio de Chimalhuacán del Estado de México. Esta enfermedad es un problema de salud pública en México, ha ocupado el primer lugar en muertes maternas por más de dos décadas ${ }^{1}$ y se presenta del 5 al $10 \%$ de todos los embarazos. ${ }^{2}$ Maffesoli, Morín, Maturana, Varela, Figuereido y Boff son los autores que dieron el soporte teórico. Se realizaron entrevistas a profundidad a dieciséis mujeres usuarias del Hospital General Chimalhuacán durante el embarazo. El análisis de los datos se realizó considerando los principios de Orlandi. ${ }^{3}$ Del análisis del discurso surgieron dos categorías: Auto-eco-socio-organización de la cotidianidad y Creando Vida en la cotidianidad. La primera categoría con 3 subcategorías: Tiempo, espacio y movimiento, identidad y, solidaridad. La segunda categoría, con dos subcategorías: Corporalidad-Subjetividad y trastrocamiento de la salud. En las consideraciones finales se enfatiza que la cotidianidad de las mujeres que presentan pre-eclampsia durante el embarazo se caracteriza por ser un fenómeno complejo, autónomo: auto-organizador, auto-productor, autodeterminado, relacional, irreductible, dialógico, recursivo, hologramático, dinámico, cambiante (neguentrópico), homeostático, sinérgico; se trastoca, se vive con identidades múltiples y solidarias frente a las adversidades. Con estos hallazgos se presentan algunos indicativos importantes para construir modelos singulares, sensibles y culturalmente específicos para el cuidado de estas mujeres.

" Estos resultados son parte de la Tesis Doctoral titulada "Cotidianidad y Cuidado de las Mujeres que presentan preeclampsia”, Programa Doctoral en Ciencias de Enfermería de la Escuela de Post Grado de la Universidad Nacional de Trujillo. Perú, en convenio con la Universidad de Celaya Guanajuato, Campus Salvatierra.

-Maestra en Ciencias de Enfermería- Profesora Asociada "C" de T.C. de la ENEO-UNAM, Doctoranda en Ciencias de Enfermería de la Escuela de Post Grado de la Universidad Nacional de Trujillo. E-mail: angelestorres2007@gmail.com •-Doctora en Enfermería de la Universidad Federal de Rio de Janeiro - Brasil. Profesora Principal de la Facultad de Enfermería de la Universidad Nacional de Trujillo. E-mail: flor_mlvm@yahoo.com ..Doctora en Ciencias de Enfermería la Universidad Federal de Rio de Janeiro - Brasil y Doctora en Administración de las Organizaciones por la Facultad de Contaduría y Administración de la UNAM. Profesora-Investigadora Titular de Tiempo Completo de la Universidad Autónoma Metropolitana-Xochimilco. E-mail: ajimenez@correo.xoc.uam.mx

Fecha de Recibido: 29 JUNiO 2012

FeCHA DE ENVIADO: 6 JULIO 2012

FECHA DE ACEPTADO: 30 AgOSTO 2012 


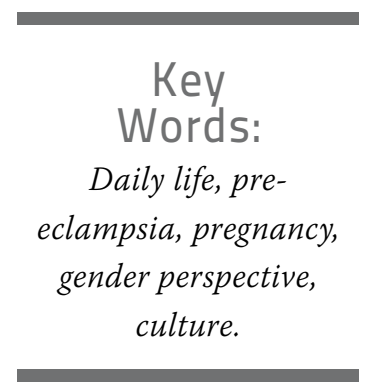

Abstrac

This qualitative, descriptive and exploratory study has the objective of describing and analyzing the everyday life of women with pre-eclampsia in a semi-urban community in the municipality of Chimalhuacán, state of Mexico. Pre-eclampsia is a public health problem in Mexico which has occupied the first place in maternal deaths over two decades, 1 and which occurs in 5 to 10\% of all pregnancies. 2 Maffesoli Morin, Maturana, Varela, Figueiredo and Boff works provide theoretical foundations for this study. Depth interviews were conducted to sixteen women attending the Chimalhuacán General Hospital during their pregnancies. The data analysis was performed considering the principles of Orlandi.3 From the discourse analysis, two categories emerged: Auto-eco-socio-organization of the daily life, and creating life in the everyday. The first category included three subcategories: time, space and movement, and identity and solidarity. The second category included two subcategories: corporeality-subjectivity, and health disruption. The final considerations of the everyday emphasizes women's pre-eclampsia as complex, autonomous, self-organizing, self-produced, self-determined, relational, irreducible, dialogic, recursive, hologramatic, dynamic, changing (negentropy), homeostatic, synergistic, and as living with multiple identities and endurance against adversity. The study findings can help design indicative singular, sensitive and culturally especific models for these women.

\section{INTRODUCCIÓN}

Este estudio permite explorar espacios de vida, espacios vividos, tiempos, actividades diarias, vínculos societales, historias de vida, organización, adaptación, la lucha diaria por la vida, el sentimiento de pertenencia a un lugar, la singularidad, identidad, solidaridad, subjetividad y aspectos socioculturales en que viven su día a día las mujeres embarazadas que cursan con preeclampsia.

El interés de esta investigación surge a raíz de mi experiencia como enfermera y docente del área obstétrica, ligada a la promoción de la salud y a la prevención de las enfermedades intercurrentes durante el embarazo, parto y puerperio; etapas de la vida de las mujeres que están íntimamente vinculadas a su cotidianeidad y al contexto sociocultural en el que se desarrolla el proceso de gestación.
Fue durante las prácticas de obstetricia con las mujeres embarazadas con pre-eclampsia, donde pude identificar que algunas de estas pacientes experimentaban momentos de estrés, de angustia, tristeza y desesperación por el temor de perder a su bebé, por el desconocimiento de la enfermedad y lo que estaba pasando con su salud, por el temor de perder su trabajo, la situación económica limitada en la que vivían, por descuidar actividades que consideran deberes de acuerdo a su género y situación conyugal como los quehaceres que dejaban al estar internadas, el cuidado de la familia y el esposo, en particular hijas e hijos pequeños que dejan al cuidado de otros, sin saber que estaban bien, habían comido o ido a la escuela.

Así mismo pude observar algunos aspectos socioculturales que li- mitaban el cuidado: algunas no llevaban control prenatal, asistían al hospital hasta llegado el momento del nacimiento. Otras consultaban solas o en compañía de sus hijos menores. Algunas tenían que recorrer largas distancias caminando para acudir a la cita; un gran número no asistía para la revisión a pesar de sentir malestares por no contar con el consentimiento del esposo y otras lo hacían hasta que el marido pudiera acompañarlas al no permitirles salir de sus casas.

Entre otros aspectos las caracterizaban, el tener que enfrentar solas el embarazo por ser madres solteras abandonadas por su pareja; vivir situaciones de violencia física, psicológica y económica aún cursando los últimos días del embarazo; algunas eran golpeadas, agredidas verbal y sexualmente; aún con malestares tenían que 
continuar haciendo las actividades propias del hogar: lavar, planchar, cocinar, cuidar de los hijos y del marido, entre otras cosas.

Por tales situaciones, comprendí que la cotidianidad en que se desarrolla el embarazo de cada mujer tiene que ver con sus emociones, miedos, angustias, enojos, frustraciones, estrés, además de la violencia y la inequidad en la que viven, lo que hace que un proceso fisiológico como lo es el embarazo, pueda sufrir alteraciones que propicien rupturas y trastoquen la normalidad de este proceso. Por tales situaciones, entendí que el cuidado de la salud durante la gestación está íntimamente ligado a su cotidianidad y a los diversos contextos en los que se desarrolla el curso de la gestación: biológico, psicológico, social, laboral, educativo, cultural, espiritual, ecológico, económico, político y/o histórico.

La cotidianidad de la mujer con pre-eclampsia ha sido poco explorada por las y los proveedores del cuidado de la salud desde el punto de vista temático y desde un abordaje metodológico cualitativo donde se describan dimensiones de aspectos subjetivos de corporalidad, social y cultural.

La investigación en este campo se ha desarrollado fundamentalmente desde la perspectiva del investigador, ${ }^{4}$ enfocada en la atención biomédica, en el campo epidemiológico y se desconoce la perspectiva de la mujer con pre-eclampsia y otro tipo de necesidades, particularmente en mujeres de condición socioeconómica desfavorable que es la de mayor riesgo de complicaciones y muerte. ${ }^{5,6}$

La pre-eclampsia y la enfermedad hipertensiva durante el emba- razo se han constituido un problema de injusticia social e inequidad en salud. La OMS estima que del 15 al 29\% de las muertes maternas en un año, fueron por causa de estos padecimientos. ${ }^{7}$ En América Latina y el Caribe personifican el $25.7 \%{ }^{7}$ En México constituyen el $28.4 \%$, han ocupado el primer lugar en las estadísticas de morbilidad y mortalidad materna por más de dos décadas y se presentan en aproximadamente del 5 al $10 \%$ de todos los embarazos; ${ }^{2}$ en el 2010 se presentaron 992 muertes maternas y un $25 \%$ (248/992) correspondieron a estas causas. $^{8}$

En el Estado de México los procesos hipertensivos durante el embarazo ocupan el primer lugar entre sus principales causas ${ }^{9}$ con el 20\% del total de defunciones maternas, ${ }^{10}$ cabe señalar que en el 2010 se presentaron 80 muertes en el Estado de México de las 837 registradas en el país por esta causa. ${ }^{11}$ En el Hospital Materno-Infantil de Chilmahuacán perteneciente al estado de México, se han presentado muertes maternas por esta causa, pues son una población de alto riesgo por sus contextos desfavorables de pobreza, falta de acceso a los servicios prenatales, niveles educativos básicos, servicios públicos precarios, problemas de inseguridad, violencia e inequidad de género, de acuerdo con los estudios de comunidad de la zona realizados durante mi práctica docente con estudiantes de enfermería de la Escuela Nacional de Enfermería y Obstetricia.

Etimológicamente el termino cotidianidad se deriva del latín "quitidianus", "de quotidie", que quiere decir cada día, diariamente. $\mathrm{Su}$ adjetivo hace referencia a algo que sucede o se hace habitualmente, rutinariamente", algo que es "natural”. Sus sinónimos son: habitual, acostumbrado, usual, frecuente, ordinario, común. ${ }^{12}$ Y el Descriptor de Ciencias de la salud ${ }^{13}$ la define como "actividades cotidianas básicas de cuidado personal, tales como vestirse, deambular, o comer", es algo que pasa todos los días.

El referencial teórico del trastocamiento de la salud en el contexto de la cotidianidad y del cuidado se basó en Maffesoli, Morín, Maturana, Varela, Figuereido y Boff, autores que dieron el fundamento teórico, a los resultados encontrados. Maffesoli orientó el estudio a partir del análisis sociológico posmoderno de la vida social con base en las categorías: adaptación a la vida, la duplicidad, el silencio, lo imaginario, la solidaridad y la proxemia (estar juntos sensible) como fuente de socialidad (vínculo social, sentimiento de pertenencia); así como en sus proposiciones que señalan que existe una relación dialéctica entre el orden y el desorden, algo que enfatiza la vida en movimiento, la circulación de bienes (renovación, bienestar social, la conquista de lo movible) y la metamorfosis de las identidades, el paso de lo estático y lo dinámico a través de la dialógica (dos lógicas antagónicas, pero complementarias) el orden/desorden, sujeto/objeto, hombre/mujer. Morín dio el soporte a los principios teóricos del pensamiento complejo: dialógico, recursión, emergencia y auto-eco-socio-organización. Maturana y Varela, para las bases conceptuales de la autopoiesis en el proceso de crear vida; Boof, para entender los fundamentos del cuidado y sus resonancias y Figueiredo para fundamentar la corporalidad. 
El pensamiento de estos autores, permitieron visualizar los fenómenos emergentes que se presentan en el contexto de la cotidianidad y cuidado de las mujeres embarazadas que presentan pre-eclampsia y que da evidencia de los cambios que produce una enfermedad emergente, como lo es la pre-eclampsia, que como fenómeno emergente, trastoca la salud, la organización, la cotidianidad y la vida misma.

Es fundamental que la mujer que ha sido diagnosticada con preeclampsia asista a control prenatal, conozca las dificultades o factores facilitadores para enfrentar su cotidianidad con acciones de autocuidado: alimentación adecuada, descanso y de ocio, evitar estrés, asistir a consultas, percibir señales corporales de malestar, buscar ayuda y movilizar redes sociales en caso necesario, de tal forma que el proceso de cuidado sea esencial y estructure su día a día. Para ello es primordial conocer la cotidianidad y el cuidado de las mujeres con pre-eclampsia y analizar las dimensiones resultantes, cómo las incorporan a su vida diaria, qué cambios presentan, qué situaciones y dificultades enfrentan, y cómo se organizan para afrontar el trastocamiento de la salud en sus diversos contextos. Desde esta perspectiva, se planteó la siguiente pregunta de investigación. ¿Cómo es la cotidianidad y el cuidado de las mujeres que presentan pre-eclampsia de una comunidad semi-urbana del Municipio de Chimalhuacán del Estado de México?

\section{Objetivos}

Describir y analizar la cotidianidad y el cuidado de las mujeres que presentan pre-eclampsia durante el embarazo en una comunidad semiurbana del Municipio de Chimalhuacán del Estado de México.

\section{Metodología.}

Investigación descriptiva-exploratoria de abordaje cualitativo desarrollada en los domicilios de las mujeres embarazadas en una comunidad semiurbana del Municipio de Chimalhuacán del Estado de México y en los servicios de hospitalización y de terapia intensiva del Hospital General de Chimalhuacán del Instituto de Salud del Estado de México. Participaron 16 mujeres, quienes otorgaron su consentimiento informado por escrito para audio-grabar y difundir la información proporcionada. El número de participantes se definió utilizando el criterio de saturación teórica. ${ }^{14} \mathrm{La}$ recolección de datos de la información se realizó de noviembre 2011 a febrero 2012 a través de entrevistas a profundidad utilizando la técnica de bola de nieve ${ }^{15}$ y la nota de campo para registrar los discursos y el lenguaje no verbal. ${ }^{16}$ Para identificar el origen de los discursos se usó un nombre ficticio para cada participante. El procesamiento de datos fue por medio del análisis de discurso de Orlandi ${ }^{3}$ Las narraciones de las mujeres participantes fueron analizadas de acuerdo con el abordaje teórico. Las entrevistas fueron transcritas literalmente tal como fueron expresadas por los sujetos (texto EMIC), luego se realizó la reducción de los datos a texto ETIC sin perder la esencia del mensaje. Seguidamente se organizaron, clasificaron, redujeron a unidades mínimas y se clasificaron los testimonios semejantes y relevantes para identificar las categorías y subcategorías que emergieron del análisis.
Para el análisis se consideraron cuatro criterios de rigor científico: credibilidad, confiabilidad, confirmabilidad y transferibilidad. ${ }^{16} \mathrm{La}$ investigación se desarrolló considerando los aspectos éticos conforme a lo establecido en la Ley General de Salud y su reglamento en materia de Investigación, respetando los principios de confidencialidad, anonimato y privacidad. ${ }^{17}$ Se sometió a evaluación del comité de investigación y ética del Hospital General Chimalhuacán, se obtuvo el consentimiento informado de las participantes para la transcripción, análisis y publicación de los discursos y se respetó la confidencialidad de la información obtenida, manteniendo el anonimato y privacidad a través de la utilización de seudónimos.

\section{Resultados y Discusión.}

A partir de los discursos obtenidos emergieron dos categorías: La primera categoría fue Auto-eco-socio-organización, con tres: subcategorías: a) Tiempo, espacio y movimiento, $b$ ) identidad y $c$ ) solidaridad; y la 2a. Categoría: "Creando vida", con dos subcategorías: Corporalidad-Subjetividad y Trastocamiento de la salud (Ver fig. 1). En este artículo sólo se presentarán los resultados de la subcategoría, "Trastocamiento de la salud" (Ver fig. 2). Ver tabla 1 para el perfil socio demográfico de las participantes.

El pensar el proceso de crear vida como un sistema vivo desde una teorización compleja, tiene una lógica composicional dinámica en el sentido de conjugar actividades de organización-creación que configuran una dialógica antagónica y complementaria con procesos de auto-destrucción/autoconstrucción en el proceso mismo 


\section{Figura 1. Categorías empíricas que emergieron del objeto de estudio cotidianidad y cuidado de las mujeres embarazadas que presentan pre-eclampsia}

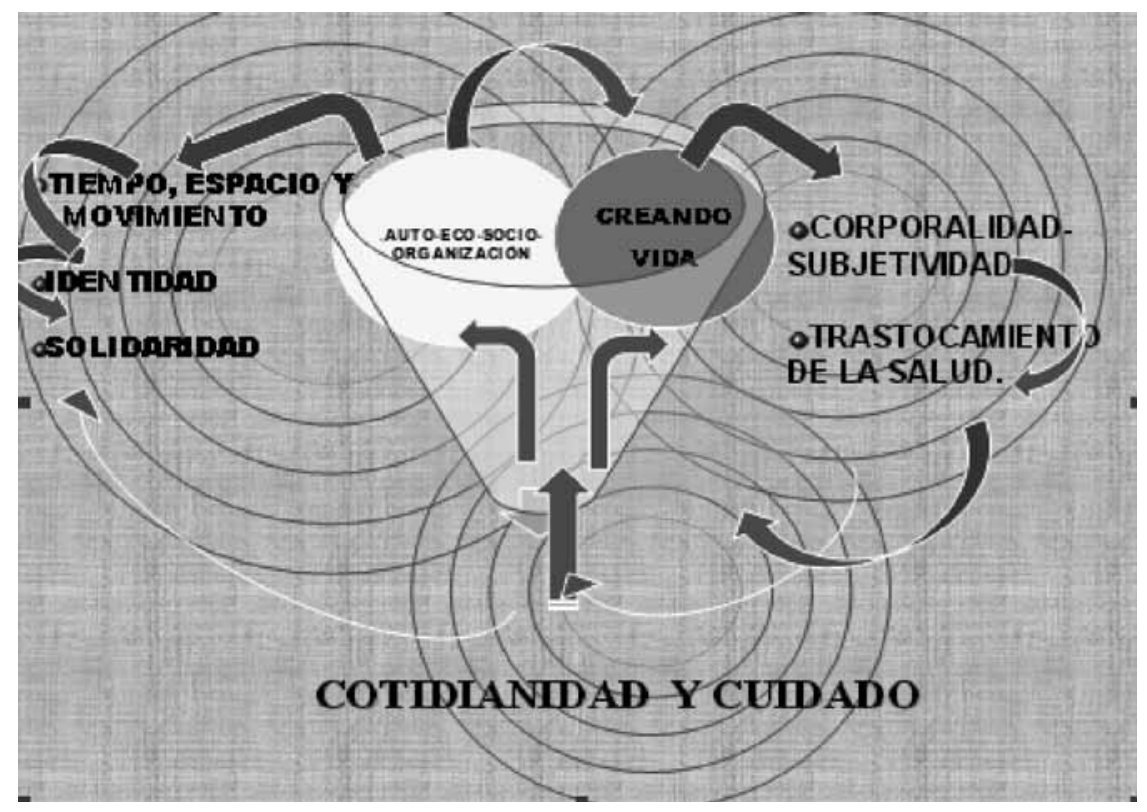

\section{Figura 2}

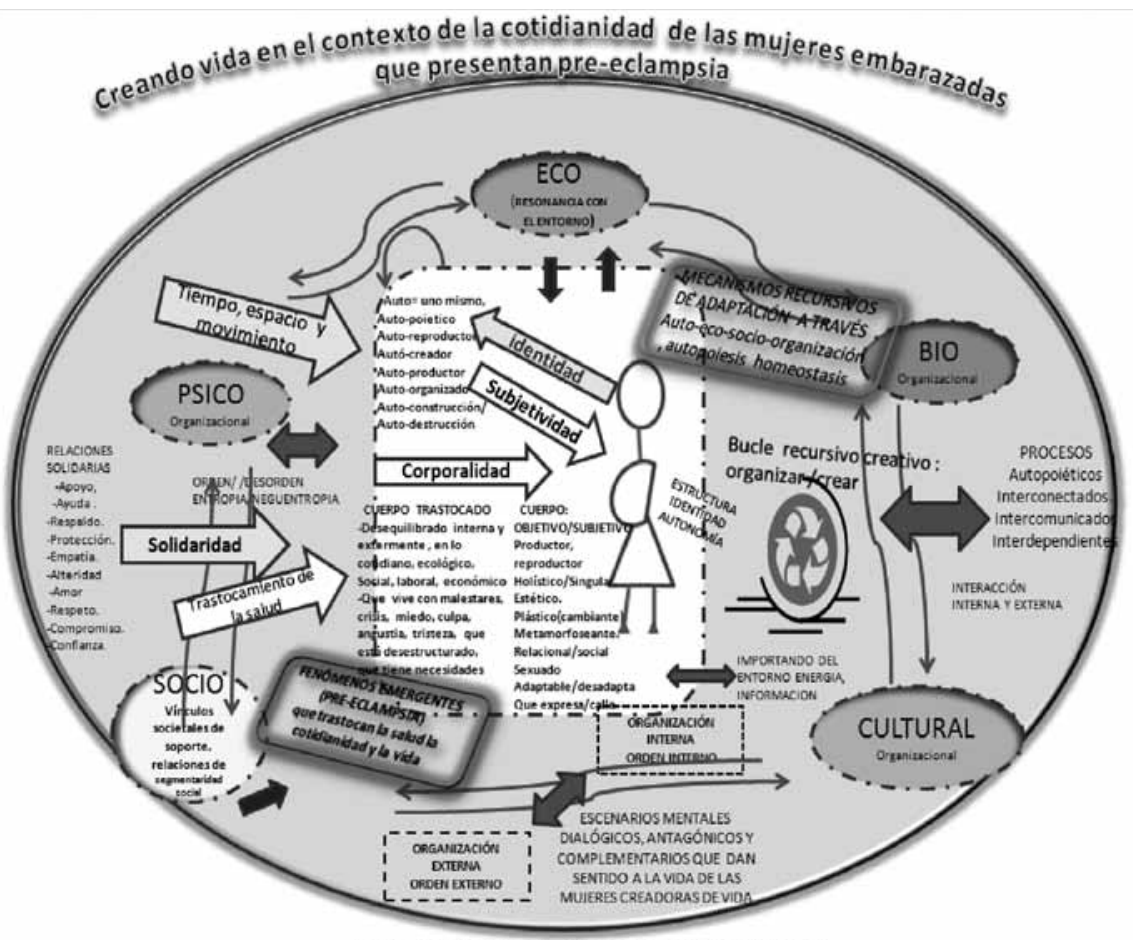

CATEGORIA CREANDO VIDA de concebir una nueva vida. En este sentido, el crear vida es un proceso que puede traer como resultado no solamente la creación sino también el trastocamiento de la salud, de la cotidianidad o la muerte misma. La vida entendida como parte de los procesos de autoconstrucción que un ser humano tiene y la enfermedad como parte de los fenómenos emergentes que alteren la salud, como lo es la enfermedad de la pre-eclampsia durante el proceso creativo del embarazo.

El crear vida hace referencia a la autopoiesis, a la auto-reproducción que tiene lugar cuando una unidad produce otra con organización similar a la de ella misma, mediante un proceso acoplado al proceso de su propia producción. Es evidente que sólo los sistemas autopoiéticos pueden auto-reproducirse, porque ellos son los únicos que se forman por un proceso de autoreproducción. ${ }^{18}$

La Corporalidad-subjetividad de las mujeres embarazadas que presentan pre-eclampsia, se refiere a la relación que tiene el cuerpo objetivo-subjetivo de la mujer embarazada que presenta pre-eclampsia con su contexto cotidiano y con el cuidado.

El trastocamiento de la salud de una mujer embarazada está relacionado con la presencia de fenómenos emergentes, dados por la enfermedad como la pre-eclampsia a partir de la cual se generan cambios en su organización interna y externa, propiciados por el estado de desorden excesivo, que altera el equilibrio de la homeostasis del cuerpo objetivosubjetivo de la mujer, desacoplando su estructura, organización e identidad, que pueden manifestarse a través de sensaciones, sentimientos, emociones y más. 
Tabla. No. 1 Perfil General de las Mujeres Entrevistadas

\begin{tabular}{|c|c|c|c|c|c|c|c|c|}
\hline Participante & $\begin{array}{l}\text { Edad } \\
\text { Años }\end{array}$ & Estudios & Ocupación & $\begin{array}{l}\text { Estado } \\
\text { civil }\end{array}$ & $\begin{array}{l}\text { Núm } \\
\text { Emb. }\end{array}$ & $\begin{array}{l}\text { SDG } \\
\text { Actul }\end{array}$ & $\begin{array}{l}\text { SDG } \\
\ln / \mathrm{pr}\end{array}$ & $\begin{array}{l}\text { Lugar } \\
\text { residencia }\end{array}$ \\
\hline 1 Marilú & 33 & $2^{\circ}$. Bachillerato & Repostera & Casada & II & 35 & 22 & $\begin{array}{l}\text { En casa } \\
\text { de su mamá }\end{array}$ \\
\hline 2 Cindy & 35 & $3^{\circ}$. Primaria & Hogar & Casada & III & 35 & 30 & $\begin{array}{l}\text { en casa } \\
\text { de su mamá }\end{array}$ \\
\hline 3 Jenny & 20 & $6^{\circ}$. Primaria & Hogar & $\begin{array}{l}\text { Unión } \\
\text { libre }\end{array}$ & II & 37.3 & $\begin{array}{l}\text { Se enteró } \\
\text { al final }\end{array}$ & $\begin{array}{l}\text { en casa } \\
\text { de su mamá }\end{array}$ \\
\hline 4 Nery & 38 & $5^{\circ}$. Primaria & Hogar & $\begin{array}{l}\text { Unión } \\
\text { libre }\end{array}$ & II & 30 & 29 & $\begin{array}{l}\text { en terreno } \\
\text { de su papá }\end{array}$ \\
\hline 5 Samanta & 18 & Preparatoria & $\begin{array}{l}\text { Prof. } \\
\text { de guardería }\end{array}$ & $\begin{array}{l}\text { Unión } \\
\text { libre }\end{array}$ & I & 32 & 30 & $\begin{array}{l}\text { en casa } \\
\text { de su mamá }\end{array}$ \\
\hline 6 Savalegui & 21 & $3^{\circ}$. Preparatoria & Hogar & Casada & I & 33 & 28 & en casa propia \\
\hline 7 Adriana & 26 & Primaria & Hogar & Casada & V & 36.4 & 35 AP & en casa propia \\
\hline $8 \mathrm{Sol}$ & 36 & $\begin{array}{l}\text { Técnica } \\
\text { en informática }\end{array}$ & Hogar & $\begin{array}{l}\text { Unión } \\
\text { libre }\end{array}$ & II & 38 & 35 & $\begin{array}{l}\text { en casa de su } \\
\text { mamá }\end{array}$ \\
\hline 9 Paula & 36 & $6^{\circ}$ Primaria & Hogar & $\begin{array}{l}\text { Unión } \\
\text { libre }\end{array}$ & IV & 35 & $22 \mathrm{AP}$ & $\begin{array}{l}\text { en casa } \\
\text { de su mamá }\end{array}$ \\
\hline 10 Ana & 31 & Preparatoria & Hogar & Casada & I & 34 & 34 & $\begin{array}{l}\text { en casa } \\
\text { de su mamá }\end{array}$ \\
\hline 11 Sarahi & 22 & $\begin{array}{l}\text { Técnica } \\
\text { en enfermería }\end{array}$ & Hogar & Casada & II & 38 & $\begin{array}{l}\text { Se enteró } \\
\text { al final }\end{array}$ & $\begin{array}{l}\text { en casa } \\
\text { de su mamá }\end{array}$ \\
\hline 12. Guadalupe & 36 & Primaria completa & $\begin{array}{l}\text { Taller } \\
\text { de costura }\end{array}$ & Casada & III & 38 & 33 & $\begin{array}{l}\text { en casa } \\
\text { de su mamá }\end{array}$ \\
\hline 13 Aurelia & 43 & Primaria completa & Hogar & Casada & VIII & 38 & 33 & en casa propia \\
\hline 14 María & 35 & Primaria completa & Hogar & Soltera & III & 38 & 24 & En casa rentada \\
\hline 15 Luna & 30 & Licenciatura & $\begin{array}{l}\text { Profesora } \\
\text { de Primaria }\end{array}$ & Casada & I & 38 & 34 & en casa propia \\
\hline 16 Estrella & 40 & Carrera Técnica & $\begin{array}{l}\text { Empleada } \\
\text { del gobierno }\end{array}$ & Casada & I & 36 & $\begin{array}{l}\text { No } \\
\text { recuerda }\end{array}$ & en casa propia \\
\hline
\end{tabular}

Las mujeres embarazadas con pre-eclampsia manifiestan su trastocamiento a través de sentimientos y emociones: angustia, culpa, temor, tristeza, preocupación, etc.

[...] "Cuando me enteré que tenía la presión alta me preocupe aunque después me sentí tranquila, no siento culpa alguna por la enfermedad, porque me cuide durante mi embarazo, pero siempre, cuando, menos lo esperas, ahi algo aparece, algo ocurre." (Ana)

\section{[...] He vivido algunos momen-} tos críticos durante el embarazo pues he tenido miedo de perder al bebé, de no hacer las cosas bien... tuve miedo y me daba tristeza. Ahora ya me siento mejor, me he cuidado (Marilú).

Ana y Marilú demuestran que inicialmente viven momentos de crisis, angustia y culpa, por el temor de perder al bebé, por el trastocamiento que produce en ellas la enfermedad de la pre-eclampsia, la cual emergió de manera inespera- da, imprevista y silenciosa. Pero antes de terminar su testimonio, ellas manifiestan que a partir de que se han cuidado pueden enfrentar la enfermedad y mantener el equilibrio, la organización y la identidad de su corporalidad-subjetividad.

Morin ${ }^{19}$ en relación al principio de emergencia señala que de las realidades organizadas emergen cualidades y propiedades nuevas (emergencias) que no son reducibles a los elementos que las componen y que retro-actúan sobre esas realidades. Las emergencias son 
definibles como "cualidades de un sistema que presentan un carácter de novedad con relación a las cualidades o propiedades de los componentes considerados aisladamente o dispuestos de forma diferente en otro tipo de sistema"

Maturana $\mathrm{H}$ y Varela $\mathrm{F}^{20}$ refieren que la "adaptación de una unidad en un medio es una consecuencia necesaria del acoplamiento estructural en ese medio". La ontogenia de un individuo es una deriva del cambio estructural con invarianza de la organización y por lo tanto con la conservación de adaptación.

\section{La subjetividad de las mujeres embarazadas que presentan pre- eclampsia se trastoca por el te- mor latente de no crear vida.}

[...] Cuando me dijeron que tenía pre-eclampsia, sentí nervios, no sé, estaba yo pensando mucho. 'Para mí la pre-eclampsia es una enfermedad mala, porque se puede morir uno. Mi preocupación cuando me dijeron que tenía preeclampsia es que nadie sabe dónde estoy, para que le informen que es lo que va a pasar conmigo. (Nery)

[...] Mi embarazo fue planeado, pero la pre-eclampsia no, ino para nada!, eso es algo que nadie lo desea, (Jenny)

Nery refiere sensaciones de angustia, soledad, abandono y aislamiento, porque se siente desamparada, sin apoyo, tiene temor a la pérdida, a la posibilidad de no poder crear vida y desconocer lo que pasará con ella.

La enfermedad puede alterar el funcionamiento psicológico y afectar la autopercepción del embarazo induciendo actitudes desfavorables ante este y generar más quejas somáticas en comparación con aquellas mujeres que el embarazo es normal. ${ }^{21}$

Con base en lo anterior se puede afirmar que el embarazo fenómeno emergente de la pre-eclampsia trastoca la salud de la mujer, produciendo alteraciones que generan crisis, angustia, sentimientos de culpa, tristeza y malestares, así como alteraciones del equilibrio cotidiano ecológico, social, cultural y económico.

\section{El trastocamiento de la salud de las mujeres embarazadas con pre-eclampsia genera cambios en la cotidianidad.}

Las mujeres embarazadas con preeclampsia refieren cambios en su cotidianidad que trastocan su corporalidad-subjetividad al generar desorden, desequilibrios en la homeostasis; así como alteraciones en la organización interna y externa en sus dimensiones físicas, sociales, culturales, ecológicas y como sus tiempos, espacios y movimientos cotidianos.

[...] Tener pre-eclampsia me ha generado algunos cambios en mi vida, pues ya no me puedo parar, no puedo caminar mucho, ya no puedes comer ciertas cosas [...] (Samanta).

[...] Le comento que mi vida diaria cambio, me siento más cansada, no puedo hacer las cosas como antes!, me daba flojera ir hacer todas las cosas, no siento ganas ni de caminar, me canso mucho, la energía que tenía ya no es igual, ya no es lo mismo, pienso que es por el bebé, hacía años que no me embarazaba, pero pienso que va terminar al nacer el bebé, creo que se me quitara todo. [...] (Nery)
Samanta y Nery describen cómo se trastoca su salud; la enfermedad les ha producido cambios físicos, que limitan el movimiento, el tránsito, la energía vital, el tipo de alimentación y la dinámica cotidiana.

[...] Mi vida diaria cambió desde que me avisaron que era pre-eclampsia, me mandaron a internar, mi esposo tiene que pedir permiso en el trabajo para venir a verme, mis hijitas están chiquitas no pueden venir. Desde que estoy internada ya me cambiaron mí comida [...] (Cindy)

[...] El tener pre-eclámpsia ha generado algunos cambios en mi vida como es que ya no cargo cosas pesadas, no puedo estar todo el día parada. Nada más me dedico al hogar y a mis quehaceres, ya no puedo como antes, me siento mal porque ya no puedo trabajar, estar aqui sentada me hacer sentir mal, pues con lo que me da mi esposo ya no nos alcanza y siento feo, pero el doctor me ha dicho que esta situación es pasajera [...] (Adriana)

Cindy y Adriana por su parte demuestran que la presencia de la pre-eclampsia les ha provocado cambios en su dinámica social y laboral al restringir sus actividades y espacios relacionales son sus compañeros de trabajo, con las hijas e hijos pequeños porque no los pueden visitar en el hospital. Les han disminuido su economía, les ha provocado problemas laborales y malestares; sin embargo, ellas tienen la idea de que el trastocamiento es transitorio y terminará cuando finalice el embarazo.

[...]Aqui en casa también tengo que estar acostada y todo eso, 
pero en casa tienes tus cosas, tu aire, tu familia que te va a ver y estás más tranquila, que si te quieres cambiar de ropa te cambias, si te quieres bañar, te bañas, tienes hambre, tienes sed, pues solo lo dices y ya van y me ayudan. [...] (Jenny).

La Sra. Jenny muestra que ha tenido cambios, en sus tiempos, espacios y movimientos cotidianos, pues al estar internada en un hospital, está confinada y restringida a las dinámicas y normas institucionales, y ello provoca que su cotidianidad también se trastoque.

Maffesolín ${ }^{22}$ al respecto, señala que existe una relación dialéctica entre el orden y el desorden, algo que enfatiza la vida, el movimiento, dando por supuesto que en la vida hay algo que no puede abarcarse, encerrarse por completo en lo instituido y en lo ordenado; serían así dos estilos de representación y de organización de la sociedad.

El trastrocamiento de la salud y de la cotidianidad propician que la mujer embarazada que presenta pre-eclampsia se auto-eco-socio-organice.

Las mujeres embarazadas con preeclampsia refieren que deben sobreponerse a pesar de las escazas fuerzas físicas y psíquicas que tienen por el trastocamiento a su corporalidad-subjetividad, así como de su salud, su cotidianidad y a su vida. Ellas asumen el rol de género que socio culturalmente la sociedad les ha asignado, en cuanto al cuidado y educación de los hijos, y tienen presente seguir realizando esas actividades para sacar esa tarea adelante, independientemente de su estado general. Ellas se auto-socio- organizan con redes de apoyo ya sea familiar, social, institucional, espiritual. Enfrentan la situación con consultas médicas, internamientos por parte de la institución, ayuda económica, acompañamientos, apoyo doméstico por parte de la familia, que es el principal apoyo que reciben. Otras formas de organizarse es a través del cuidar de sí mismas: llevan a cabo los consejos médicos, se dan masajes, crean espacios de descanso y sueño, evitan comer ciertos alimentos, se auto-miden la presión arterial, realizan actividades recreativas, y más. Los siguientes testimonios dan cuenta de ello:

[...] "Vivo con mi esposo, él me ha apoyado mucho porque mis hijas están chiquitas; pero a pesar de ser chiquitas ellas ven que van a comer y me ayudan con el quehacer de la casa. La primera vez para ir a consulta llegaba yo solita, pero cuando me dijo el doctor que tenia esta enfermedad, ya él me empezó a acompañar y me apoya". [...] (Cindy)

[...] "Trataba de no gastar mucho para que nos alcanzara para la semana, me iba a mis consultas caminando aunque está un poco retirado de mi casa el centro de salud. Me acompañaba mi niño el mayorcito y él se regresaba porque tenía que arreglarse para la escuela, y se regresaba solito. El mayorcito me apoyaba a cuidar a sus hermanitos." [...] [Paula].

[...] "Cuando me sentía mal mi hijo me sobaba mis pies y mi hija la grande me sobaba el vientre; para mí ya era insoportable mi dolor de espalda. Luego me decía tomate algo, pero nada más tocamos cama y a dormir. De hecho cuando me siento mal, mi hija me dice vámonos al doctor; le decía no, sé que, me voy a quedar ya no voy a salir. Cuando me puse mal si, se movía muy lento, por eso fui al doctor. Tengo mis hermanos y mis tías, me apoyan económicamente, me van a ver, sí me han ayudado mucho." [...] [María].

Las tres mujeres demuestran que se auto-eco-socio-organizan recibiendo apoyo familiar y soporte hospitalario. Cindy refiere el apoyo por parte del marido e hijos, de quienes recibe compañía para las consultas y apoyo en las tareas domésticas. Paula señala el apoyo por parte del hijo, quien la acompaña para las consultas y cuida de los hermanitos y finalmente María recibe apoyo por parte de sus hijos y otros familiares quienes le dan masajes, la animaban, la acompañaban al médico y le propiciaban espacios de descanso y relajación; también la alentaban a asistir al médico y a tomar sus medicamentos, además de apoyarla económicamente.

[...] "Mi presión está bien, pero porque la cuido, mi mamá me ayuda, en lo que como, no como mucha sal, no tomo coca, ni cafeína; tomo té $y$ agua, medicamentos solo el ácido fólico y el hierro". [...] (Samanta)

[...]Cuidados en este embarazo, ya no como mucha sal y tomo agua. Si me siento mal, me recuesto un rato y ya pasaba el dolor y me levanto. Convivo más con mi mamá, cuando voy a visitarla me apapacha. [...] (Sarahi)

[...] Hago los cuidados normales del embarazo, que me decía la doctora, que me tomara mi medicamento, que no comiera gra- 
sas, que hiciera ejercicio, que me mantuviera activa porque me quiso dar como depresión [...] (Sol)

[...] "Para sentirme mejor me acostaba, me relajaba, me quedaba dormida y me sentía mejor." [...] (Guadalupe)

[...]"Cuando tenía algún malestar iba a recostar un ratito y me sentía mejor. A veces si me quedaba dormida hasta media hora $y$ me levantaba. Si noto que sigo igual de mal pues al otro día voy al doctor." [...] (Aurelia)

[...]"Para estar bien y hago lo que me dicen para que nazca bien, no subo ni bajo tantas escaleras y trato de cuidarme sobre todo en lo que como" [...] (María)

[...] Me estoy tomando la presión para ver como está, cuando iba bajando me despreocupé. A veces se me olvida la enfermedad por las actividades y me siento bien gracias a los medicamentos y a la dieta que seguí [...] (Estrella).

Samanta, Sarahí, Sol, Guadalupe, Aurelia, María y Estrella, indican que se auto-eco-organizan además del soporte familiar e institucional, con el autocuidado: crean espacios para descansar y dormir, evitan comer ciertos alimentos, ingieren suficiente agua y se auto-administran sus medicamentos, realizan ejercicio, o llevan a cabo los consejos de sus médicos, controlan la energía de su cuerpo y la presión arterial, entre otras cosas más.

[... ]iPrimero Dios todo va a estar bien!, mi bebé se está moviendo bien. [...] (Adriana)

[...]Mi mamá no me pue- de ayudar pero me basta con sus bendiciones. ¡Gracias a Dios tengo unos tíos muy buenos. [...] (María)

Finalmente Adriana y María además, refieren que dentro de los soportes que reciben está el fortalecimiento espiritual con sus creencias y su fe en un ser superior.

\section{Consideraciones finales}

En este estudio, observamos que la presencia de la enfermedad de la pre-eclampsia durante el embarazo limita las funciones vitales, disminuye la energía, la oxigenación, el bienestar, el tránsito, los espacios, los tiempos de convivencia con la familia, altera las actividades diarias, interrumpe las actividades laborales y la normalidad del diario vivir.

La emergencia de enfermedades en el proceso de crear vida, trastoca la salud, la cotidianidad y la vida, propiciando que las mujeres se auto-eco-socio-organicen, favoreciendo con ello los mecanismos recursivos de adaptación, organización, autopoiesis y homeostasis. La pre-eclampsia trastoca la vida, el vivir y la corporalidad subjetiva y objetiva de la mujer embarazada haciéndola coexistir con situaciones de crisis que se manifiestan a través de sentimientos y emociones tales como: angustia, abandono, aislamiento, temor, entre otras más.

El cuerpo de una mujer embarazada que presenta pre-eclampsia tiene mecanismos recursivos que le permiten auto-eco-socioorganizarse para lograr mantener el equilibrio, la organización, la adaptación y la lucha diaria por la vida, a pesar del devenir de cambios inesperados, en su dinámica cotidiana, en sus tiempos, espacios, interacciones y vínculos societales que se ven trastocado por los fenómenos emergentes. Es un cuerpo que expresa malestares y necesidades de cuidado y que al mismo tiempo se auto-socio-ecoorganiza de manera interna a través de su corporalidad y subjetividad y en su contexto externo mediante las instituciones y de redes de apoyo (social, espiritual, económica), de sus espacios de recreación, de sueño y descanso además del tratamiento médico (medicamentos, nutrición, asistencia prenatal y asesorías para su cuidado y visitas periódicas). Se auto-socioorganizan en su contexto interno a través del cuidado de su corporalidad-subjetividad, evitando comer ciertos alimentos, siguiendo las recomendaciones del personal de salud, creando espacios de descanso, sueño y relajación a través de masajes que les dan sus hijos, se auto-miden la presión arterial, realizan actividades recreativas, $y$ más. $Y$ en su contexto externo se auto-eco-socio-organizan a través de redes de apoyo familiar, social, institucional y espiritual. Enfrentan la situación con consultas médicas, internamientos por parte de la institución, ayuda económica, acompañamiento y apoyo doméstico de la familia que es el soporte principal.

La prevención en maternidad debe ser descentrada de los aspectos biomédicos e incluir otras dimensiones, tales como las subjetivas, las socioculturales y las políticas. Se debe tener modelos de maternidad participativa donde las mujeres sean sujetos activos respecto de su propia salud, considerando que la prevención en la maternidad debe 
incluir las determinantes de salud y enfermedad de la mujer desde la cotidianidad y el cuidado así como la calidad de vida de la mujer como creadora de vida, considerando los hallazgos de esta investigación.

A partir de los hallazgos de este estudio, es necesario integrar a las prácticas preventivas de la maternidad aquello vinculado a la cotidianidad y el cuidado, como son las condiciones de vida, el trabajo doméstico y extra doméstico, educación y relación con su familia, historia de vida, sexualidad, mitos, ritos, así como explicar el lugar que ocupa la mujer creadora de vida en la sociedad desde una perspectiva compleja, no biologicista, no patriarcal.

\section{REFERENCIAS BIBLIOGRÁFICAS}

1 Avena JL, Joerin VN, Dozdor LA, Bres SA. Preeclampsia/Eclampsia. Revista de Posgrado de la VI Cátedra de Medicina [Internet]. 2007; (165):20-5. [Acceso: 01/07/2011] Disponible en: http://bit.ly/ pQJ94L.

2 OMM. Observatorio de Mortalidad Materna en México. Indicadores Nacionales y panorama epidemiológico de muertes maternas. México, D.F. Disponible en: http:// bit.ly/13fSvsn

3 Orlandi E. Análisis de discurso: principios e procedimentos. 4a, ed. Brasil: Bazuca; 2002.

4 Mendoza-Sassi R, Béria J. Utilización de los servicios de salud: una revisión sistemática sobre los factores relacionados. Cuadernos médicos sociales. 2002; 83:43-60.

5 Ali AA, Adam I. Lack of antenatal care, education, and high maternal mortality in Kassala hospital, eastern Sudan during 2005-2009. J Matern Fetal Neonatal Med. 2011;24(8):1077-8.
6 Gonzalez R, Requejo JH,Nien JK, Merialdi M, Betran AP. Chile Maternal, newborn, and Child Health Writing Group. Tackling Health Inequities in Chile: Maternal, Newborn, Infant, and Child Mortality Between 1990 and 2004. Am J Public Health 2009; 99(7):12206.

7 OMS. Causes of maternal death. Systematic review identifies main causes of maternal mortality and morbidity. Progress in Reproductive Health Research 2005; (71):5-7 Disponible en: http://bit.ly/Yf35dd

8 Freyermuth G, Luna M, Ochoa P. Mortalidad Materna en México. Numeralia 2010. CIESAS-OPSOMM. México 2011.

9 Gómez-Bravo E, Briones JC, Morales JG, Rodríguez M. Mortalidad materna en el Edo. de Méx. Rev Asoc MexMed Crit Ter Int. 2003;17(6):199-203.

10 Instituto de Salud del Estado de México. Mortalidad Materna según causa 2002-2009. México. Disponible en: http://bit.ly/X6CQYx

11 SSA. Panorama Epidemiológico e indicadores de muerte materna 2010. Reunión Nacional Programa Arranque Parejo para la Vida. México, D.F.: 2010. Disponible en: http://bit.ly/YSviq2

12 Diccionario de la Real Academia de la Lengua Española.España2012.

13 BIREME. Descriptores de Ciencias de la Salud. Biblioteca virtual de la salud. 2012.

14 Polit D, Hungler B. Investigación científica en Ciencias de la Salud. México, D.F.: McGraw-Hill; 2000.

15 Souza-Minayo MC. El desafío del conocimiento. Investigación cualitativa en salud. Sao Paulo, Brasil, Hucitec editor. 2006.

16 Ulin PR, Robinson ET, Tolley EE. Invitación a investigar. Investi- gación aplicada en salud pública: Metodos cualitativos. Pub Cient No 614. Washington DC, EUA: Family Health International, Organización Panamericana de la Salud, 2006.

17 Secretaría de Salud. Reglamento de la Ley General de Salud en materia de investigación para la salud,Titulo Segundo. De los aspectos éticos de la Investigación. Capítulo I. 1984-88. Disponible en: http://www.salud.gob.mx.

18 Maturana H y Varela F. De Máquinas a Seres vivos. Autopoiesis: la organización de lo vivo. Santiago de Chile: Universitaria; 1997.

19 Morin E. El método, 1. La naturaleza de la naturaleza. Madrid, España: Cátedra, Colecc. Teorema; 2009.

20 Maturana H y Varela F. El árbol del conocimiento. Bases biológicas del entendimiento humano. Campinas: Ed.Psy II; 1985.

21 Salvatierra V. Psicología del embarazo y sus transtornos. Barcelona, España; 1989.

22 Maffesoli M. Hipótesis sobre el Eon Posmoderno. Conferencia de Michel Maffesoli 29 de abril de 2004. Revista Faces (Venezuela) [Internet]. [1-6 pp]. Disponible en: http://bit.ly/XxqUyv 